\title{
Pulmonary Tuberculosis Complicated with Severe Thrombocytopenia with Diffuse Alveolar Hemorrhaging during Anti-tuberculosis Chemotherapy: An Autopsy Report
}

\author{
Takehiko Kobayashi ${ }^{1}$, Kazunari Tsuyuguchi ${ }^{2,3}$, Takahiko Kasai ${ }^{3,4}$ and Katsuhiro Suzuki ${ }^{1}$
}

\begin{abstract}
:
Tuberculosis may be accompanied by various hematological abnormalities during treatment, and occasionally thrombocytopenia is also noted. Most cases of thrombocytopenia in tuberculosis are moderate, and there are few reports about thrombocytopenia with diffuse alveolar hemorrhaging (DAH) in pulmonary tuberculosis. We describe the case of an 82-year-old man with pulmonary tuberculosis and tuberculous pleurisy. $\mathrm{He}$ underwent anti-tuberculosis chemotherapy; during tuberculosis treatment, he experienced DAH due to fulminant thrombocytopenia. Thrombocytopenia is a common finding in patients with pulmonary tuberculosis, but the laboratory findings should be carefully evaluated. Thrombocytopenia during anti-tuberculosis chemotherapy can cause life-threatening DAH.
\end{abstract}

Key words: pulmonary tuberculosis, pulmonary alveolar bleeding, thrombocytopenia

(Intern Med 57: 3285-3288, 2018)

(DOI: 10.2169/internalmedicine.0207-17)

\section{Introduction}

Tuberculosis may be accompanied by various hematological abnormalities during treatment, and thrombocytopenia is also occasionally experienced. Thrombocytopenia tends to cause bleeding in severe cases. However, it is rare that it accompanies diffuse alveolar hemorrhaging (DAH). DAH is a clinical diagnosis characterized by diffuse radiographic alveolar infiltrates and hemoptysis, which is usually accompanied by respiratory failure (1).

We herein report the case of an 82-year-old man with pulmonary tuberculosis and tuberculous pleurisy. He underwent anti-tuberculosis chemotherapy; during tuberculosis treatment, the patient experienced DAH due to fulminant thrombocytopenia.

\section{Case Report}

An 82-year-old man presented with a 1-month history of appetite loss followed by slow progressive dyspnea in December 2015. The patient was an ex-smoker (7 pack-year) and had received donepezil hydrochloride $(50 \mathrm{mg} /$ day orally) for Alzheimer's disease. The patient had symptoms of worsening dyspnea from February 2016.

On an examination, he was alert and did not appear to be overtly unwell. His body temperature was $37.2^{\circ} \mathrm{C}$. Pulse oximetry showed an oxygen saturation of $94 \%$ at room pressure. His breath sounds were decreased at the left-sided lung base. There were no signs of lower extremity deep-venous thrombosis and palpable lymph nodes. Chest X-ray films showed a left-sided pleural effusion. Chest computed tomography (CT) revealed left-sided pleural effusion and centrilobular nodules in all fields(Fig. 1a). Blood and serologic examinations revealed a normal white blood cell count

\footnotetext{
${ }^{1}$ Department of Internal Medicine, National Hospital Organization, Kinki-Chuo Chest Medical Center, Japan, ${ }^{2}$ Department of Infectious Diseases, National Hospital Organization, Kinki-Chuo Chest Medical Center, Japan, ${ }^{3}$ Clinical Research Center, National Hospital Organization, Kinki-Chuo Chest Medical Center, Japan and ${ }^{4}$ Department of Pathology, National Hospital Organization, Kinki-Chuo Chest Medical Center, Japan

Received: September 13, 2017; Accepted: April 15, 2018; Advance Publication by J-STAGE: July 6, 2018

Correspondence to Dr. Kazunari Tsuyuguchi, tsuyuguchi@kch.hosp.go.jp
} 


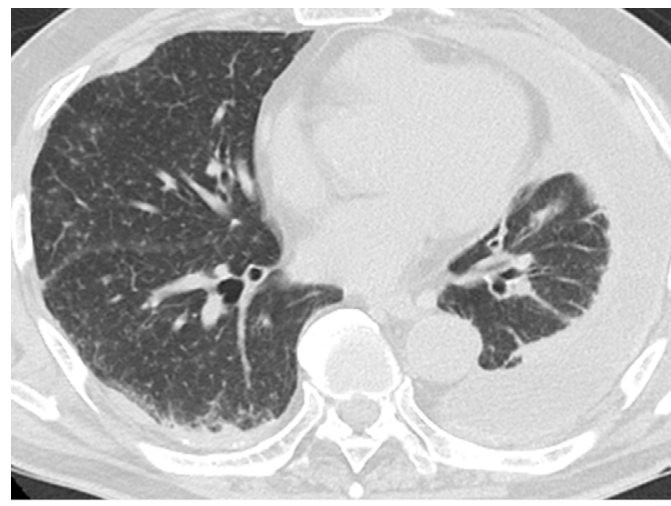

a)Day1

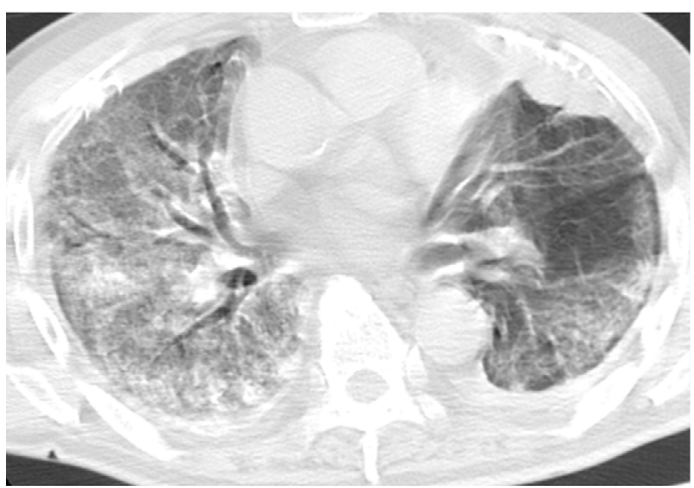

c) Day 61

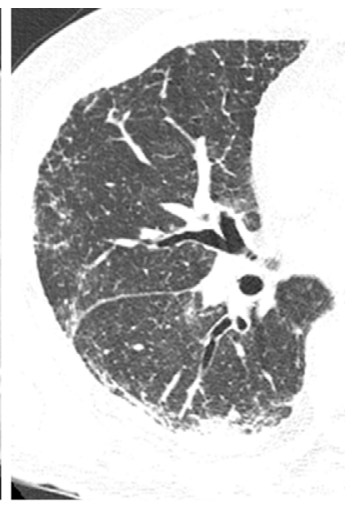

b) Day 47

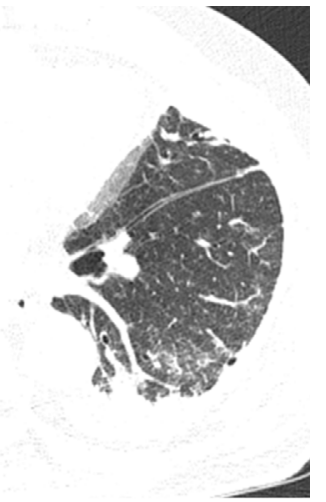

Figure 1. Computed tomography (CT) of the chest: (a) lower lung fields on day 1 showing left-sided pleural effusion and centrilobular nodules in all fields; (b) high-resolution CT on day 47 showing bilateral improvement of centrilobular nodules and decreased pleural effusion; (c) CT of the lower lung fields on day 61 showing bilateral worsening of the alveolar spaces in the lower lung fields.

$(5,800$ cells $/ \mu \mathrm{L})$ and moderately increased $\mathrm{C}$-reactive protein (CRP) $(3.57 \mathrm{mg} / \mathrm{dL})$, normal hemoglobin $(14.8 \mathrm{~g} / \mathrm{dL})$, and normal thrombocyte $\left(29.3 \times 10^{4}\right.$ cells $\left./ \mu \mathrm{L}\right)$ levels. An examination of the left-sided pleural fluid revealed exudative pleural effusion with increased lymphocytes $(95 \%)$ and high adenosine deaminase levels (123.0 U/L), whereas smears, cultures, and cytology of the pleural fluid were negative. Mycobacterium tuberculosis was isolated from sputum samples. The patient was diagnosed with pulmonary $M$. tuberculosis and M. tuberculosis pleurisy. He was referred to Kinki-Chuo Chest Medical Center, a 385-bed center in Southern Osaka that specializes in pulmonary disease for pulmonary $M$. tuberculosis treatment.

After receiving a 3-drug regimen [rifampicin (RFP) 600 $\mathrm{mg} /$ day, isoniazid (INH) $300 \mathrm{mg} /$ day, and ethambutol (EB) $1,000 \mathrm{mg} /$ day] according to the patient's weight for 2 months, his general condition was stable, and his symptoms of dyspnea resolved gradually. His sputum smear was negative on hospital day 28, and chest CT on hospital day 47 revealed that the left-sided pleural effusion had ameliorated, and the centrilobular nodules had gradually decreased in number (Fig. 1b). However, the patient complained of fatigue and presented with skin rash with pruritus over the lumbar and neck region on hospital day 50. The thrombocyte count was 220,000 cells $/ \mu \mathrm{L}$ on that day. Although all medication was stopped because the skin rash was suspected to be drug-induced, progressive fatigue, dyspnea, and massive hemoptysis appeared with intraoral bleeding on hospital day 61.

His body temperature was $38.0^{\circ} \mathrm{C}$, and pulse oximetry showed an oxygen saturation of $81 \%$ at room pressure. Coarse crackles were heard in the bilateral lung fields without wheezing. Hepatic splenomegaly was not noted. Blood and serologic examinations revealed fulminant thrombocytopenia (thrombocyte count of 1,000 cells $/ \mu \mathrm{L}$ ), and coagulation marker levels were normal (prothrombin time: $1.38 \mathrm{sec}-$ onds; prothrombin time-international normalized ratio: 1.16; activated partial thromboplastin time: 28 seconds). An arterial blood gas analysis with 10-L oxygen administration revealed marked hypoxemia [partial pressure of oxygen $\left(\mathrm{PaO}_{2}\right)$ $64.6 \mathrm{mmHg}$. Chest $\mathrm{CT}$ revealed bilateral and progressive multiple areas of subpleural and peribronchial patchy consolidation (Fig. 1c).

The patient was administered methylprednisolone $(1 \mathrm{mg} /$ $\mathrm{kg} /$ day) and transfused with multiple units of platelets. Another regimen of anti-tuberculosis chemotherapy (Streptomycin $0.75 \mathrm{~g} /$ day and Levofloxacin $500 \mathrm{mg} /$ day intravenously) was started, but his pulmonary condition deteriorated progressively, and he passed away on hospital day 65 .

Drug-induced lymphocyte stimulation tests (DLSTs) of 


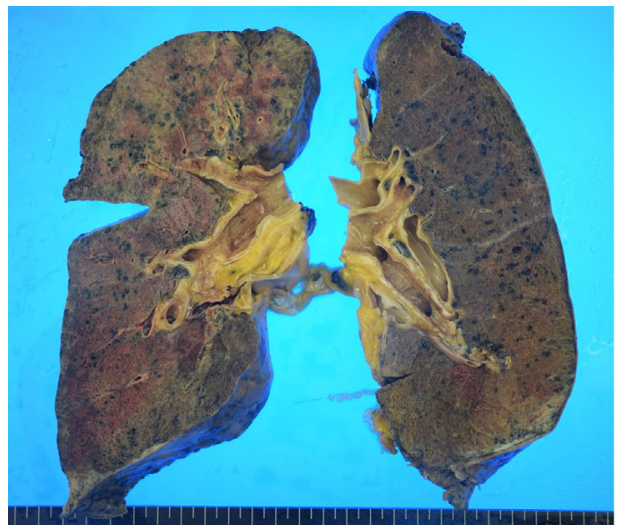

(a)

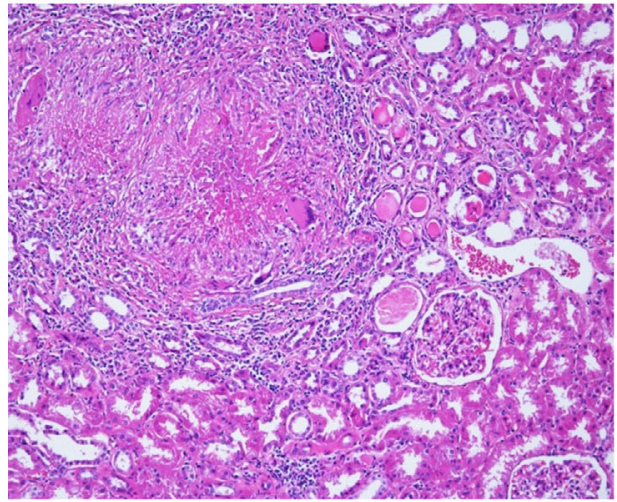

(c)

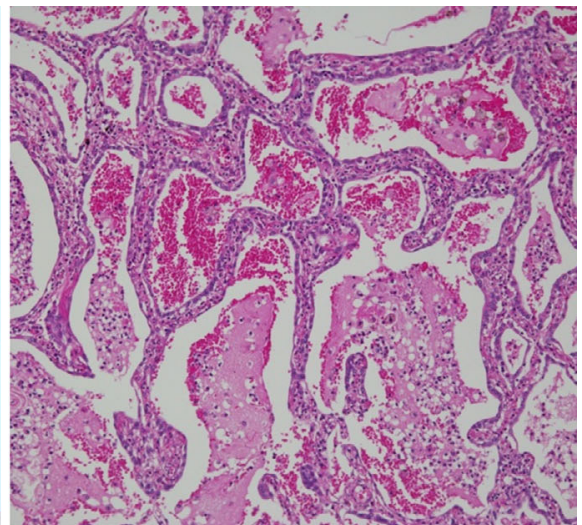

(b)

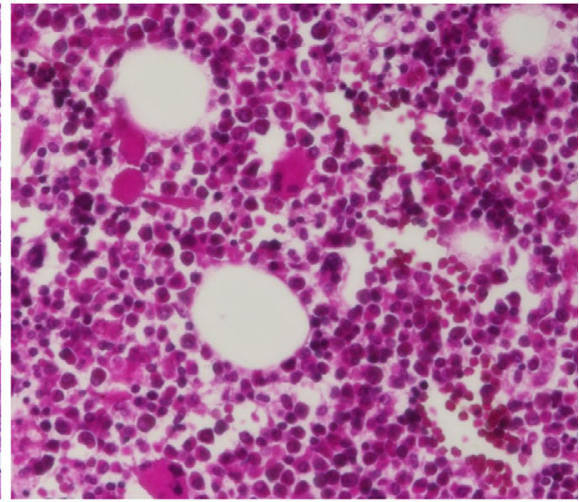

(d)

\begin{abstract}
Figure 2. (a) Both lungs revealed diffuse bleeding lesions. (b) Sample from lungs [Hematoxylin and Eosin $(H \& E)$ staining, $\times 400$ ]; the alveolar spaces are diffusely filled with blood, and foci of hemosiderin-laden macrophages are present. Mild interstitial inflammation and type II pneumocyte hyperplasia are observed. (c) Samples from the kidney (H\&E staining, $\times 400)$; in the kidney, the glomeruli did not reveal thrombotic microangiopathy but did show granulomas with necrosis. (d) Samples from the bone marrow (H\&E staining, $\times 400$ ); The hyperplastic bone marrow revealed no epithelioid cell granulomas.
\end{abstract}

RFP and INH were performed after his death (INH: positive; RFP: negative). An autopsy was performed after obtaining permission from the family. Autopsy specimens of the lung revealed DAH comprising intraalveolar hemorrhaging, hemosiderin-laden macrophages, and type II pneumocyte hyperplasia (Fig. 2a and b). The glomeruli had no microthrombi but demonstrated well-formed epithelioid cell granulomas with central necrosis, indicating miliary tuberculosis (Fig. 2c). The hyperplastic bone marrow showed increased numbers of megakaryocytes but no epithelioid cell granulomas of military tuberculosis. (Fig. 2d).

\section{Discussion}

The present case highlighted two important clinical issues: severe thrombocytopenia can present during tuberculosis treatment, and DAH can occur as a complication of thrombocytopenia in pulmonary tuberculosis.

First, regarding the issue of severe thrombocytopenia, several reports of thrombocytopenia during anti-tuberculosis treatment have been documented (2-4). Some reports have described the mechanisms of thrombocytopenia being due to the activity of tuberculosis itself. The mechanisms underlying immune thrombocytopenia have been considered to be due to the suppression of $\mathrm{T}$ cells derived from tuberculosis and the production of antiplatelet antibodies, and the conversion of latent antibody into platelets might therefore have caused the manifestation of thrombocytopenia $(5,6)$. However, the patient in this case did not have any systemic symptoms, such as neurological symptoms or kidney failure; therefore, thrombotic thrombocytopenic purpura was not suspected. Idiopathic thrombocytopenic purpura is diagnosed by exclusion, but it is also necessary to perform a bone marrow puncture. However, bone marrow aspiration was not performed in the case as the clinical course of the patient was rapid and deteriorated with acute respiratory failure.

Drug-induced thrombocytopenia may also occur because of allergic reactions to INH or RFP during anti-tuberculosis chemotherapy. We performed a DLST of INH and RFP in the present study, but the efficacy of DLST against antituberculosis drugs in thrombocytopenia is unclear (7). The clinical course and autopsy findings in this patient suggested 
that the anti-tuberculosis treatment might have been related to the thrombocytopenia.

Although George et al. found that most reports of druginduced thrombocytopenia have not provided evidence supporting a definite or probable causal relationship between the disease and the drug in their systemic review (8), and the exact mechanism underlying drug-induced thrombocytopenia remains unclear. However, some reports have hypothesized that the mechanism is related to anti-tuberculosis treatment. It has been rationalized that RFP-dependent antibodies bind to thrombocytes and cause its increased destruction. INH may also induce disturbances in the hematological system (4).

As mentioned above, the present case highlighted the fact that DAH as a complication of thrombocytopenia can also occur in pulmonary tuberculosis patients. Significant decreases in the platelet count can cause superficial oral and nose bleeding but seldom cause DAH. In this case, the patient died due to the complication of respiratory failure following pulmonary alveolar bleeding. Few reports in the literature have described DAH as a complication of thrombocytopenia. Only three previous reports have documented thrombocytopenia complicated with idiopathic thrombocytopenia purpura following DAH (9-11). The autopsy in the present case showed that there were no findings of complication with vasculitis. Although mycobacteriological and radiological examinations confirmed that the patient's pulmonary tuberculosis had been successfully treated, inflammation caused by the infection may have caused an increase in the vascular permeability, which further promoted bleeding with thrombocytopenia.

In conclusion, we herein report a rare case of DAH in the setting of pulmonary tuberculosis. Some adverse effects may be experienced during tuberculosis treatment, but cases of DAH induced by severe thrombocytopenia during tuberculosis treatment are rare. Thrombocytopenia is a common finding in clinical practice and is occasionally associated with life-threatening bleeding complications. We report this case to raise clinical awareness of the rare but potentially fatal adverse effects of DAH-induced severe thrombocytopenia during tuberculosis treatment.
The authors state that they have no Conflict of Interest (COI).

\section{Financial Support}

The authors are partially supported by a grant from National Hospital Organization Respiratory Network, Japan.

\section{References}

1. Frankel SK, Cosgrove GP, Fischer A, Meehan RT, Brown KK. Update in the diagnosis and management of pulmonary vasculitis. Chest 129: 452-465, 2006.

2. Inegbenebor E, Saad N, Leelasinjaroen P, Khatib R. A case of immune thrombocytopenia associated with pulmonary tuberculosis. Chest 146: 180A, 2014.

3. Hashiguchi N, Furuyama K, Kim M, Hirose N. A case of pulmonary tuberculosis complicated with severe thrombocytopenia during treatment. Kekkaku 87: 345-349, 2012 (in Japanese, Abstract in English).

4. Yakar F, Yildiz N, Yakar A, Kilicaslan Z. Isoniazid- and rifampicin-induced thrombocytopenia. Multidiscip Respir Med 8: $13,2013$.

5. Jurak SS, Aster R, Sawaf H. Immune thrombodytopenia associated with tuberculosis. Clin Pediatr 22: 318-319, 1983.

6. Al-Majed SA, Al-Momen AK, Al-Kassimi FA, Al-Zeer A, Kambal AM, Baaqil H. Tuberculosis presenting as immune thrombocytopenia purpura. Acta Haematol 94: 135-138, 1995.

7. Suzuki Y, Miwa S, Shirai M, et al. Drug lymphocyte stimulation test in the diagnosis of adverse reactions to antituberculosis drugs. Chest 134: 1027-1032, 2008.

8. George JN, Raskob GE, Shah SR, et al. Drug-induced thrombocytopenia: a systematic review of published case reports. Ann Intern Med 129: 886-890, 1998.

9. Uchiyama M, Hattori A, Tanaka T, et al. Acute idiopathic thrombocytopenic purpura complicated with diffuse alveolar hemorrhage in an elderly patient. Intern Med 48: 1449-1452, 2009.

10. Das S, Cherian S, Hamarneh W, et al. Diffuse alveolar hemorrhage secondary to idiopathic thrombocytopenic purpura; an extremely rare presentation. Chest 142: 469A, 2012.

11. Hashmi HR, Venkatram S, Diaz-Fuentes G. A case report of an elderly woman with thrombocytopenia and bilateral lung infiltrates: a rare association between diffuse alveolar hemorrhage and idiopathic thrombocytopenic purpura. Medicine (Baltimore) 94: e $2235,2015$.

The Internal Medicine is an Open Access journal distributed under the Creative Commons Attribution-NonCommercial-NoDerivatives 4.0 International License. To view the details of this license, please visit (https://creativecommons.org/licenses/ by-nc-nd/4.0/).

(C) 2018 The Japanese Society of Internal Medicine Intern Med 57: 3285-3288, 2018 